\section{Gold-Plated CNTs Used as Photoacoustic and Photothermal Imaging Agents and Laser-Ablation Therapeutic Agents}

The photothermal (PT) and photoacoustic (PA) properties of carbon nanotubes (CNTs) make them promising candidates for deep-tissue imaging and treatment of tumors and infections. The possibility of toxicity, however, is a concern. In contrast, gold nanoparticles, such as gold nanoshells, gold nanorods, and gold nanocages, have minimal toxicity and show near-infrared absorption higher than CNTs. Recently, an interdisciplinary team led by J.-W. Kim at the University of Arkansas, Fayetteville, and V.P. Zharov at the University of Arkansas for Medical Sciences (UAMS), Little Rock, combined the advantages of gold nanoparticles with CNT properties, including mechanical flexibility, large surface area, small diameter, and the ability to cluster, to enhance $\mathrm{PA}$ and PT responses.

As reported in the August 23 online publication of Nature Nanotechnology (DOI: 10.1038/NNANO.2009.231), Kim, Zharov, and co-researchers E.I. Galanzha of UAMS and also of Saratov State University in Saratov, Russia, E.V. Shashkov of UAMS and also of Prokhorov General Physics Institute, Moscow, and H.-M. Moon of the University of Arkansas fabricated goldplated CNTs (GNTs) by depositing a thin Au film (2-8 nm thick) onto single-walled CNTs with diameter of $\sim 1.5 \mathrm{~nm}$. The GNTs are water soluble, well dispersed, and rodshaped with average dimensions of $100 \mathrm{~nm} \pm 36.7 \mathrm{~nm}$ in length and $11 \mathrm{~nm} \pm$ $4.1 \mathrm{~nm}$ in diameter. Optical spectral analysis showed that the GNTs have a transverse plasmon adsorption in the visible spectral range similar to gold nanospheres and a longitudinal resonance peak in the NIR similar to gold nanorods. Plasmon responses for GNTs in the NIR were 85-150 times higher than those for CNTs at the same concentration. PT images of individual GNTs showed high contrast even at extremely low laser fluences in the range of $1-10 \mathrm{~mJ} \mathrm{~cm}^{-2}$ at $850 \mathrm{~nm}$. Increasing the pulse energy resulted in increased image contrast and bubble formation, demonstrating the possibility of controlled, localized cell damage with limited damage to surrounding cells.

Live cells were exposed to concentrations of GNTs ranging over $0.05-0.5 \mathrm{mg} / \mathrm{ml}$. The researchers observed no adverse cell viability or toxicity effects after exposure for 10 days. The researchers said that these preliminary results imply minimal toxicity for GNTs.

The researchers then used GNTs to target lymphatic endothelial cells (LECs line the internal surface of lymphatic vessels and are in direct contact with immunerelated cells, tumor cells, bacteria, and other cells present in the lymph flow). GNTs were conjugated with an antibody specific to an LEC receptor, such as LYVE-1, delivered to a mouse lymphatic system, and exposed to a laser pulse using an integrated intravital microscope. Non-invasive diagnostics through PA/PT imaging were performed with low laser energy, followed by therapy-ablating individual cells-with increased laser energy. In addition, the researchers demonstrated PA detection and PT ablation of individual GNT-labeled tumor cells in lymph nodes in vivo.

The researchers said, "We are currently investigating the potential of GNTs for molecular detection and eradication of metastasis in the sentinel lymph nodes and real-time tracking of GNTs in ear and skin animal vasculatures, as well as an in vivo toxicity study of GNTs on animal models."

STEVEN TROHALAKI

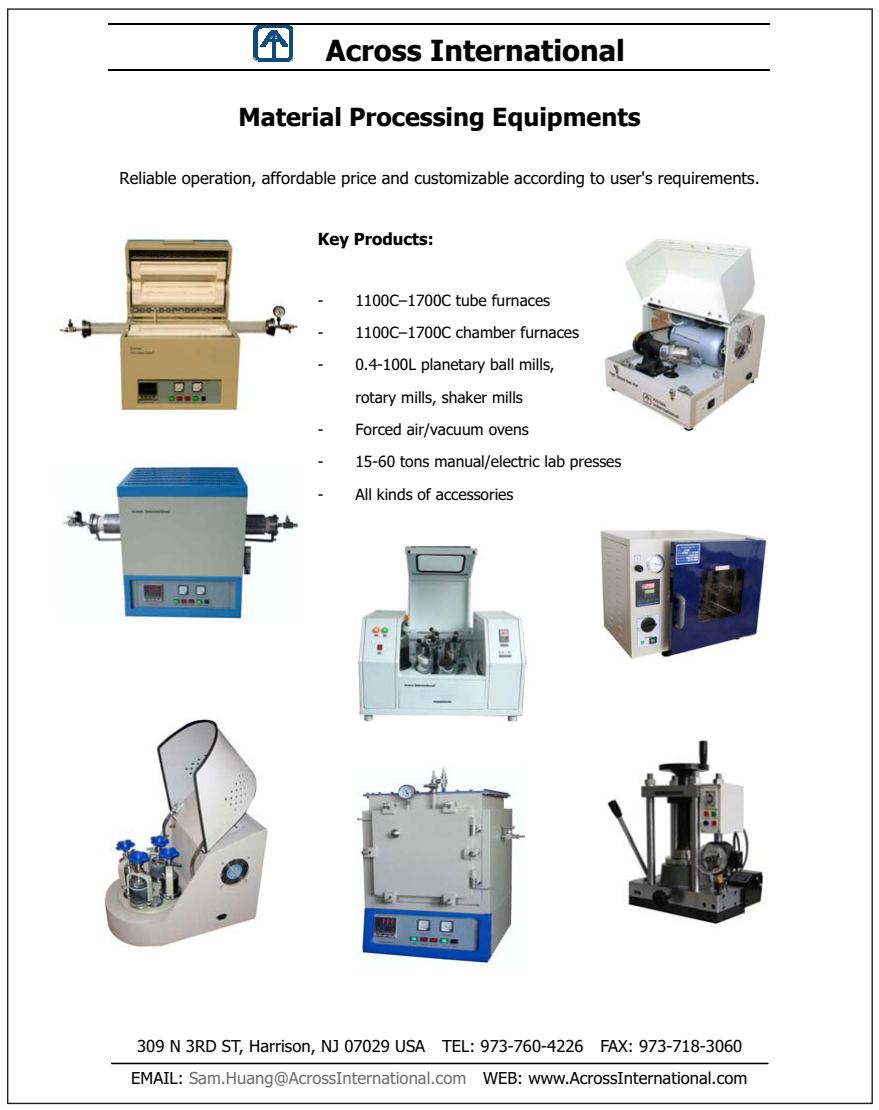

\title{
JA N S
}

Cryogen Free Probe Stations

- $<5 \mathrm{~K}-650 \mathrm{~K}$ cryocooler based systems, using no liquid cryogens

Fully vibration isolated sample mounting stage for sub-micron stability

Two to six probes, DC to $67 \mathrm{GHz}$ frequency range, with fiber optic option

Zoom optics with camera, monitor and full system integration

- Complete packages including probe station, temperature controller, optics, and vacuum pump

LHe and $\mathrm{LN}_{2}$ cooled, and room temperature probe stations also available

\section{Janis Research Company}

2 Jewel Drive Wilmington, MA 01887 USA

TEL +1 978 657-8750 FAX +1978 658-0349 sales@janis.com Visit our website at WWW.janis.com 